\title{
On Fuzzy Soft Perfectly Disconnected Spaces
}

\author{
Rajarshi Chatterjee \\ Trivenidevi Bhalotia College \\ (Affiliated To Kazi Nazrul University), India.
}

\begin{abstract}
The aim of this paper is to introduce and study the concept of fuzzy soft perfectly disconnected spaces on fuzzy soft topological spaces are introduced and several characterizations are discussed.
\end{abstract}

Keywords: fuzzy soft nowhere dense set ,fuzzy soft regular open set ,fuzzy soft perfectly disconnected space , fuzzy soft weakly Baire spaces, fuzzy soft extremely disconnected space

\section{Introduction:}

In 1965, L.A. Zadeh [14] introduced the concept of fuzzy sets as a new approach for modeling uncertainities In 1968,C.L.Chang [2 ] introduced the concept of fuzzy topological spaces. The paper of Chang paved the way for the subsequent tremendous growth of the numerous fuzzy topological concepts Perfectly disconnected space in classical topology was defined and studied by Eric K. Van Douwen [4 ].The concept of fuzzy extremally disconnected spaces was defined and studied by G.Balasubramaniam [3]. Molodtsov [6] initiated the theory of soft sets as a new mathematical tool for dealing with uncertainities which traditional mathematical tools cannot handle. He has shown several applications of this theory in solving any practical problems in economics, engineering, social sciences, medical science etc. Later other authors like Maji et al. [5] have further studied the theory of soft sets and used this theory to solve some decision making problems. The concept of fuzzy soft nowhere dense sets and Fuzzy soft weakly Baire space was introduced and studied by E.Poongothai and S.Divyapriya[7 ,8]. The concept of fuzzy perfectly disconnected spaces in topological spaces was introduced by G.Thangaraj and S.Murugannathan [ 9 ]. In this paper the notion of fuzzy soft perfectly disconnected space is introduced and several characterizations of fuzzy soft perfectly disconnected spaces are established.

\section{PRELIMINARIES}

In section 2 we have given some basic definitions and notion are self-contained.

\section{Definition 2.1[5]:}

The fuzzy soft set $F_{\phi} \in F \mathrm{~S}(U, E)$ is said to be null fuzzy soft set and it is denoted by $\phi$, if for all $e \in E, F(e)$ is the null fuzzy soft set0 of $U$, where $\overline{0}(x)=0$ for all $x \in U$.

\section{Definition 2.2 [5]:}

$\operatorname{Let} F E \in F S(U, \mathrm{E}) \operatorname{and} F E(e)=\overline{1}$ all $e \in E$, where $\overline{1}(x)=1$ for all $x \in U$.Then $\mathrm{F}_{\mathrm{E}}$ is called absolute fuzzy soft set. It is denoted by $\bar{E}$.

\section{Definition 2.3 [ 5]:}

A fuzzy soft set $F_{A}$ is said to be a fuzzy soft subset of a fuzzy soft set $G_{B}$ over a common universe $U$ if $A \subseteq B$ and $F_{A}(e) \subseteq G_{B}$ (e) for all $e \in A$, i.e., if $\mu^{\mathrm{e}} F_{A}(x) \leq \mu^{\mathrm{e}} G_{B}(x)$ for all $x \in U$ and for all $e \in E$ and denoted by $F_{A} \subseteq G_{B}$.

\section{Definition 2.4[5]:}

Two fuzzy soft sets $F_{A}$ and $G_{B}$ over a common universe $\mathrm{U}$ are said to be fuzzy soft equal if $F_{A}$ is a fuzzy soft subset of $G_{B}$ of $G_{B}$ is a fuzzy soft subset of $F_{A}$.

\section{Definition 2.5[5]:}

The union of two fuzzy soft sets $F_{A}$ and $G_{B}$ over the common universe $\mathrm{U}$ is the fuzzy soft set $H C$, defined by $H_{C}(e)$ $=\mu_{H_{\mathrm{C}}}^{e}=\mu_{F_{\mathrm{A}}}^{e} \cup \mu_{G_{B}}^{e}$ for all $e \in E$, where $C=A \cup B$. Here we write $H_{C}$ $=F_{A} \breve{V} G_{B}$

\section{Definition 2.6[6]:}

Let $F_{A}$ and $G_{B}$ be two fuzzy soft set, then the intersection of $F_{A}$ and $G_{B}$ is a fuzzy soft set $H_{C}$, defined by $(e)=\mu_{F_{\mathrm{C}}}^{e}=\mu_{F_{\mathrm{A}}}^{e} \cap \mu_{G_{\mathrm{B}}}^{e}$ for all $e \in E$, where $C=A \cap B$. Here we write $H_{C}=F_{A} \bar{\wedge} G_{B}$

\section{Lemma 2.1[1]:}

For a family $\mathrm{A}=\left\{\lambda_{\alpha}\right\}$ of fuzzy sets of a fuzzy space $\mathrm{X}, V\left(\operatorname{cl}\left(\lambda_{\alpha}\right.\right.$ )$) \leq \operatorname{cl}\left(V\left(\left\{\lambda_{\alpha}\right)\right.\right.$. In case A is a finite set, $V\left(\operatorname{cl}\left(\lambda_{\alpha}\right)\right)=\operatorname{cl}\left(V\left(\left(\lambda_{\alpha}\right)\right)\right.$ . Also $V\left(\operatorname{int}\left(\lambda_{\alpha}\right)\right) \leq \operatorname{int}\left(V\left(\lambda_{\alpha}\right)\right)$.

\section{Definition 2.7[10]:}

Let $F_{A} \in \mathrm{FS}(U, \mathrm{E})$ be a fuzzy soft set. Then the complement of $F_{A}$, denoted by $F_{A}^{C}$,defined by

$F_{A}^{C}(e)=\left\{\begin{array}{cc}\overline{1}-\mu_{F \mathrm{~A}}^{e}, & \text { if } e \in \mathrm{A} \\ \overline{1}, & \text { if } \notin \notin \mathrm{A}\end{array}\right\}$ 


\section{Definition 2.8[10]:}

Let $\psi$ be the collection of fuzzy soft sets over $U$. Then $\psi$ is called a fuzzy soft topology on $U$ if $\psi$ satisfies the following axioms:

(i) $\phi, \bar{E}$ belong to $\psi$.

(ii) The union of any number of fuzzy soft sets in $\psi$ belongs to $\psi$.

(iii) The intersection of any two fuzzy soft sets $\psi$ belongs to $\psi$. The triplet $(U, E, \psi)$ is called a fuzzy soft topological space over $\mathrm{U}$. The members of $\psi$ are called fuzzy soft open sets in $U$ and complements of them are called fuzzy soft closed sets in $\mathrm{U}$.

\section{Definition 2.9[10]:}

The union of all fuzzy soft open subsets of $F_{A}$ over $(U, E$, ) is called the interior of $F_{A}$ and is denoted byint $f^{\mathrm{s}}\left(F_{A}\right)$.

\section{Proposition 2.1[10]:}

$\operatorname{int}^{\mathrm{fs}}\left(F_{A} \bar{\wedge} G_{B}\right)=\operatorname{int}^{f \mathrm{~S}}\left(F_{A}\right) \widetilde{\Lambda} i n t^{f s}\left(G_{B}\right)$.

\section{Definition 2.10 [1]:}

Let $F_{\mathrm{A}} \in \mathrm{FS}(U, E)$ be a fuzzy soft set. Then the intersection of all closed sets, each containing $F_{\mathrm{A}}$, is called the closure of $F_{A}$ and is denoted by $\mathrm{cl}^{\mathrm{fs}}\left(F_{A}\right)$.

\section{Remarks 2.11 [11]:}

(1) For any fuzzy soft set $F_{A}$ in a fuzzy soft topological space $(U, E, \psi)$, it is easy to see that $\left(\left(F_{A}\right)\right)^{\mathrm{c}}=\operatorname{int}^{f s}\left(F_{A}^{C}\right)$ and $\left(\operatorname{int}^{f \mathrm{~s}}\right.$ $\left.\left(F_{A}\right)\right)^{\mathrm{c}}=c l^{\mathrm{f}}\left(F_{A}^{C}\right)$.

(2) For any fuzzy soft $F_{A}$ subset of a fuzzy soft topological space $(U, E, \psi)$ we define the fuzzy soft subspace topology on $F_{A}$ by $K_{D} \in \psi_{\mathrm{F}_{\mathrm{A}}}$ if $K_{D}=F_{A} \nearrow_{\wedge} G_{B}$ for some $G_{B} \epsilon \psi$.

(3) For any fuzzy soft $H_{C}$ in $F_{A}$ fuzzy soft subspace of a fuzzy soft topological space, we denote to the interior and closure of $H_{C}$ in $F_{A}$ by $i n t_{F_{A}}^{f s}\left(H_{C}\right)$ and $c l_{F_{A}}^{f s}\left(H_{C}\right)$, respectively.

\section{Definition2.12[8]:}

A fuzzy soft set $\mathrm{F}_{\mathrm{A}}$ in a FSTS $(U, E, \psi)$ is called a Fuzzy Soft Nowhere Dense set if there exist no non-zero fuzzy soft open set $G_{B}$ in $(\mathrm{U}, \mathrm{E}, \psi)$ such that $G_{B}<\mathrm{cl}^{\mathrm{fs}}\left(\mathrm{F}_{\mathrm{A}}\right)$. ie $)$, int ${ }^{\mathrm{fs}} \mathrm{cl}^{\mathrm{fs}}\left(\mathrm{F}_{\mathrm{A}}\right)=0$.

\section{Definition 2.13[ 8]}

A fuzzy soft set $\mathrm{F}_{\mathrm{A}}$ in a $\operatorname{FSTS}(U, E, \psi)$ is called fuzzy soft dense if there exist no fuzzy soft closed set $G_{B}$ in $(U, E, \psi)$ such that $\mathrm{F}_{\mathrm{A}}<G_{B}<1$. ie) $\operatorname{cl}^{\mathrm{fs}}\left(\mathrm{F}_{\mathrm{A}}\right)=1$.

\section{Definition 2.14[ 8 ]:}

Let $(U, E, \psi)$ be a fuzzy soft topology . A fuzzy soft set $\mathrm{F}_{\mathrm{A}}$ in $(U, E$,$) is called fuzzy soft first category. If \mathrm{F}_{\mathrm{A}}=\mathrm{V}_{i=1}^{\infty}\left(F_{A_{i}}\right)$ where $\left(F_{A_{i}}\right)$ 's are fuzzy soft nowhere dense sets in $(U, E, \psi)$. Any other fuzzy soft set in $(U, E$,) is said to be of fuzzy soft second category.

\section{Definition 2.15[ 9 ]:}

A fuzzy soft set $\mathrm{F}_{\mathrm{A}}$ in a FSTS (U,E, $\psi$ ) is called a fuzzy soft $\sigma$ - boundary set, if $\mathrm{F}_{\mathrm{A}}=\mathrm{V}_{i=1}^{\infty}\left(\mathrm{G}_{\mathrm{B}_{\mathrm{i}}}\right)$, where $\mathrm{G}_{\mathrm{B}_{\mathrm{i}}}=\operatorname{cl}^{\mathrm{fs}}\left(\mathrm{F}_{\mathrm{A}_{\mathrm{i}}}\right)$ $\wedge\left(1-\mathrm{F}_{\mathrm{A}_{\mathrm{i}}}\right)$ and $\left(\mathrm{F}_{\mathrm{Ai}}\right)^{\prime} \mathrm{s}$ are fuzzy soft regular open sets in (U,E, $\psi$ ) (short form fuzzy soft regular open sets, fsros).

\section{Definition 2.16[9]:}

A fuzzy soft set $\mathrm{F}_{\mathrm{A}}$ in a Fsts $(\mathrm{U}, \mathrm{E}, \psi)$ is called a fuzzy soft pre $\mathrm{F}_{\sigma}-$ set, if $\mathrm{F}_{\mathrm{A}}=\mathrm{V}_{i=1}^{\infty}\left(\mathrm{G}_{\mathrm{B}_{\mathrm{i}}}\right)$, where $\left(\mathrm{G}_{\mathrm{B}_{\mathrm{i}}}\right)$ ' s are fuzzy soft pre closed sets in $(\mathrm{U}, \mathrm{E}, \psi)$.

\section{FUZZY SOFT PERFECTLY DISCONNECTED SPACES.}

\section{Definition 3.1:}

If for any two non-zero Fuzzy Soft Sets $F_{A}$ and $G_{B}$ are defined on $U$ with $F_{A} \leq 1-G_{B}, c^{f s}\left(F_{A}\right) \leq 1-c^{f s}\left(G_{B}\right)$, in Fuzzy Soft Topological Spaces $(\mathrm{U}, \mathrm{E}, \psi)$ is called a fuzzy soft perfectly disconnected space.

\section{Example 3.1.1:}

The fuzzy soft sets $F_{E}, G_{E}, H_{E}, L_{D}$, are defined on $U$ as follows.

$$
\begin{aligned}
& \mathrm{F}_{\mathrm{A}}: X \rightarrow[0,1] \text { defined as } \mathrm{F}_{\mathrm{A}}(\mathrm{a})=0.4 ; \mathrm{F}_{\mathrm{A}}(\mathrm{b})=0.7 ; \mathrm{F}_{\mathrm{A}}(\mathrm{c})=0.5 \text {; } \\
& \mathrm{G}_{\mathrm{B}}: \mathrm{X} \rightarrow[0,1] \text { defined as } \mathrm{G}_{\mathrm{B}}(\mathrm{a})=0.5 ; \mathrm{G}_{\mathrm{B}}(\mathrm{b})=0.6, \mathrm{G}_{\mathrm{B}}(\mathrm{c})=0.6 \text {; } \\
& \mathrm{H}_{\mathrm{c}}: \mathrm{X} \rightarrow[0,1] \text { defined as } \mathrm{H}_{\mathrm{c}}(\mathrm{a})=0.6 ; \mathrm{H}_{\mathrm{c}}(\mathrm{b})=0.6 ; \mathrm{H}_{\mathrm{c}}(\mathrm{c})=0.5 \text {; } \\
& \mathrm{J}_{\mathrm{E}}: \mathrm{X} \rightarrow[0,1] \text { defined as } \mathrm{J}_{\mathrm{E}}(\mathrm{a})=0.3 ; \mathrm{J}_{\mathrm{E}}(\mathrm{b})=0.5 ; \mathrm{J}_{\mathrm{E}}(\mathrm{c})=0.4 \text {. } \\
& \mathrm{L}_{\mathrm{D}}: \mathrm{X} \rightarrow[0,1] \text { defined as } \mathrm{L}_{\mathrm{D}}(\mathrm{a})=0.4 ; \mathrm{L}_{\mathrm{D}}(\mathrm{b})=0.4 ; \mathrm{L}_{\mathrm{D}}(\mathrm{c})=0.2 \text {. } \\
& \text { Now } T=\left\{0, F_{A}, G_{B}, H_{c},\left(F_{A} \vee G_{B}\right),\left(F_{A} \vee H_{c}\right),\left(G_{B} \vee H_{c}\right),\left(F_{A} \wedge G_{B}\right)\right. \text {, } \\
& \left(F_{A} \wedge H_{c}\right),\left(G_{B} \wedge H_{c}\right), F_{A} \vee\left(G_{B} \wedge H_{c}\right), G_{B} \wedge\left(F_{A} \vee H_{c}\right), H_{c} \vee\left(F_{A} \wedge G_{B}\right), \\
& \left.\mathrm{F}_{\mathrm{A}} \vee \mathrm{G}_{\mathrm{B}} \vee \mathrm{H}_{\mathrm{c}}, 1\right\} \text {. Then } \mathrm{H}_{\mathrm{c}} \leq 1-\mathrm{L}_{\mathrm{D}}, \mathrm{cl}^{\mathrm{fs}}\left(\mathrm{H}_{\mathrm{c}}\right) \leq 1-\mathrm{cl}^{\mathrm{fs}}\left(\mathrm{L}_{\mathrm{D}}\right) \text {, in }(\mathrm{U}, \mathrm{E}, \psi) \text {. }
\end{aligned}
$$

Hence $(\mathrm{U}, \mathrm{E}, \psi)$ is a fuzzy soft perfectly disconnected space.

\section{Proposition 3.1}

If $(\mathrm{U}, \mathrm{E}, \psi)$ is a Fuzzy soft pre dense set and $\mathrm{F}_{\mathrm{A}} \leq 1-\mathrm{G}_{\mathrm{B}}$ for any two non-zero Fuzzy soft sets $F_{A}$ and $G_{B}$ defined on $U$ then $\mathrm{cl}^{\mathrm{fs}}\left(\mathrm{F}_{\mathrm{A}}\right) \neq 1$ and $\mathrm{cl}^{\mathrm{fs}}\left(\mathrm{G}_{\mathrm{B}}\right) \neq 1$ in FSTS $(\mathrm{U}, \mathrm{E}, \psi)$.

\section{Proof:}

Suppose that $F_{A} \leq 1-G_{B}$ any two non-zero Fuzzy Soft Sets $F_{A}$ and $\mathrm{G}_{\mathrm{B}}$ defined on $\mathrm{U}$ then $(\mathrm{U}, \mathrm{E}, \psi)$ is a Fspds, we have $\left(\mathrm{F}_{\mathrm{A}}\right) \leq 1$ $\mathrm{cl}^{\mathrm{fs}}\left(\mathrm{G}_{\mathrm{B}}\right)$, in FSTS $(\mathrm{U}, \mathrm{E}, \psi)$. If $\mathrm{cl}^{\mathrm{fs}}\left(\mathrm{F}_{\mathrm{A}}\right)=1$, then $1 \leq 1-\mathrm{cl}^{\mathrm{fs}}\left(\mathrm{G}_{\mathrm{B}}\right)$ it implies that $\mathrm{cl}^{\mathrm{fs}}\left(\mathrm{G}_{\mathrm{B}}\right)=0$.That is $\mathrm{cl}^{\mathrm{fs}}\left(\mathrm{G}_{\mathrm{B}}\right)=0$ in $(\mathrm{U}, \mathrm{E}, \psi)$, if $\mathrm{G}_{\mathrm{B}}=$ 0 , a contradiction to $\mathrm{G}_{\mathrm{B}} \neq 0$. If $\mathrm{cl}^{\mathrm{fs}}\left(\mathrm{G}_{\mathrm{B}}\right)=1$ then $\mathrm{cl}^{\mathrm{fs}}\left(\mathrm{F}_{\mathrm{A}}\right) \leq 1-1$ $=0$. That is $\operatorname{cl}^{\mathrm{fs}}\left(\mathrm{F}_{\mathrm{A}}\right)=0$. If $\mathrm{F}_{\mathrm{A}}=0$, which is contradiction to 
$\mathrm{F}_{\mathrm{A}} \neq 0$, thus ,if $\mathrm{F}_{\mathrm{A}} \leq 1-\mathrm{G}_{\mathrm{B}}$, then $\mathrm{cl}^{\mathrm{fs}}\left(\mathrm{F}_{\mathrm{A}}\right) \neq 1$ and $\mathrm{cl}^{\mathrm{fs}}\left(\mathrm{G}_{\mathrm{B}}\right) \neq 1$ in Fuzzy Soft Topological Spaces (U,E, $\psi$ ).

\section{Proposition 3.2:}

If $\mathrm{F}_{\mathrm{A}}$ and $\mathrm{F}_{\mathrm{A}} \leq 1-\mathrm{G}_{\mathrm{B}}$ is a fuzzy soft closed set in fuzzy soft pre dense sets (U,E, $\psi$ ), then there exists a Fuzzy soft open sets $\mathrm{H}_{\mathrm{c}}$ in $(\mathrm{U}, \mathrm{E}, \psi)$. Such that $\mathrm{F}_{\mathrm{A}} \leq \mathrm{H}_{\mathrm{C}} \leq 1-\mathrm{G}_{\mathrm{B}}$.

\section{Proof:}

Let $F_{A} \leq 1-G_{B}$ and $F_{A}$ is a Fuzzy soft closed sets in Fuzzy soft pre dense sets $(\mathrm{U}, \mathrm{E}, \psi)$. Then $\mathrm{cl}^{\mathrm{fs}}\left(\mathrm{F}_{\mathrm{A}}\right) \leq 1-\mathrm{cl}^{\mathrm{fs}}\left(\mathrm{G}_{\mathrm{B}}\right)$ in FSTS $(\mathrm{U}, \mathrm{E}, \psi) . \mathrm{F}_{\mathrm{A}}$ is a Fuzzy soft closed sets in $(\mathrm{U}, \mathrm{E}, \psi)$, we have $\mathrm{cl}^{\mathrm{fs}}\left(\mathrm{F}_{\mathrm{A}}\right)=1$, and $\mathrm{F}_{\mathrm{A}} \leq 1-\mathrm{cl}^{\mathrm{fs}}\left(\mathrm{G}_{\mathrm{B}}\right)$, then $1-\mathrm{cl}^{\mathrm{fs}}\left(\mathrm{G}_{\mathrm{B}}\right) \leq 1-\mathrm{G}_{\mathrm{B}}$, in $(\mathrm{U}, \mathrm{E}, \psi)$.Therefore, $\mathrm{F}_{\mathrm{A}} \leq 1-\mathrm{cl}^{\mathrm{fs}}\left(\mathrm{G}_{\mathrm{B}}\right) \leq 1-\mathrm{G}_{\mathrm{B}}, \mathrm{H}_{\mathrm{c}}=1-\mathrm{cl}^{\mathrm{fs}}\left(\mathrm{G}_{\mathrm{B}}\right)$ .Then $\mathrm{H}_{\mathrm{C}}$ is a Fuzzy soft open set in $(\mathrm{U}, \mathrm{E}, \psi)$, Hence $\mathrm{F}_{\mathrm{A}} \leq \mathrm{H}_{\mathrm{C}} \leq$ $1-\mathrm{G}_{\mathrm{B}}$ in $(\mathrm{U}, \mathrm{E}, \psi)$, where $\mathrm{H}_{\mathrm{C}} \in T$.

\section{Proposition 3.3 :}

IF $G_{B}$ and $F_{A} \leq 1-G_{B}$ is a fuzzy soft closed set in fuzzy soft pre dense sets (U,E, $\psi$ ), then there exists a Fuzzy soft closed sets $\mathrm{L}_{D}$ in $(\mathrm{U}, \mathrm{E}, \psi)$ such that $\mathrm{F}_{\mathrm{A}} \leq \mathrm{L}_{\mathrm{D}} \leq 1-\mathrm{G}_{\mathrm{B}}$.

\section{Proof :}

Suppose that $F_{A} \leq 1-G_{B}$ and $G_{B}$ is a Fscs in $(U, E, \psi)$ is a Fuzzy soft pre dense sets, we have $\operatorname{cl}^{\mathrm{fs}}\left(\mathrm{F}_{\mathrm{A}}\right) \leq 1-\operatorname{cl}^{\mathrm{fs}}\left(\mathrm{G}_{\mathrm{B}}\right)$, then $\mathrm{F}_{\mathrm{A}} \leq$ $\mathrm{cl}^{\mathrm{fs}}\left(\mathrm{G}_{\mathrm{B}}\right) \leq 1-\mathrm{G}_{\mathrm{B}}$. Let $\mathrm{L}_{\mathrm{D}}=\mathrm{cl}^{\mathrm{fs}}\left(\mathrm{F}_{\mathrm{A}}\right)$. Then $\mathrm{L}_{\mathrm{D}}$ is a Fuzzy soft closed sets in (U,E, $\psi)$.

\section{Proposition 3.4:}

If $F_{A} \leq 1-G_{B}$ for any two FSS $F_{A}$ and $G_{B}$ defined on $U$, in a Fuzzy soft pre dense sets (U,E, $\psi$ ), then there exist a Fuzzy soft open sets $\mathrm{H}_{\mathrm{c}}$ in $(\mathrm{U}, \mathrm{E}, \psi)$. Such that int ${ }^{\mathrm{fs}} \mathrm{cl}^{\mathrm{fs}}\left(\mathrm{F}_{\mathrm{A}}\right) \leq \mathrm{H}_{\mathrm{C}} \leq 1-\mathrm{cl}^{\mathrm{fs}}$ $\left[\operatorname{int}^{\mathrm{fs}}\left(\mathrm{G}_{\mathrm{B}}\right)\right]$ and int ${ }^{\mathrm{fs}}\left(\mathrm{G}_{\mathrm{B}}\right)$ is not a fuzzy soft dense set in $(\mathrm{U}, \mathrm{E}, \psi)$.

\section{Proof:}

Let $\mathrm{F}_{\mathrm{A}} \leq 1-\mathrm{G}_{\mathrm{B}}$ in FSTS $(\mathrm{U}, \mathrm{E}, \psi)$. Then $\mathrm{cl}^{\mathrm{fs}}\left(\mathrm{F}_{\mathrm{A}}\right) \leq \mathrm{cl}^{\mathrm{fs}}\left(1-\mathrm{G}_{\mathrm{B}}\right)$ and hence $\operatorname{cl}^{\mathrm{fs}}\left(\mathrm{F}_{\mathrm{A}}\right) \leq 1-\operatorname{int}^{\mathrm{fs}}\left(\mathrm{G}_{\mathrm{B}}\right)$. Since $\mathrm{cl}^{\mathrm{fs}}\left(\mathrm{F}_{\mathrm{A}}\right)$ is a fuzzy soft closet set in FSTS (U,E, $\psi$ ), By prop 3.2, there exist a fuzzy soft open set $\mathrm{H}_{\mathrm{C}}$ in $(\mathrm{U}, \mathrm{E}, \psi)$. Such that $\mathrm{cl}^{\mathrm{fs}}\left(\mathrm{F}_{\mathrm{A}}\right) \leq \mathrm{H}_{\mathrm{C}} \leq[1-$ $\left.\operatorname{int}^{\mathrm{fs}}\left(\mathrm{G}_{\mathrm{B}}\right)\right]$. Then , int ${ }^{\mathrm{fs}}\left[\mathrm{cl}^{\mathrm{fs}}\left(\mathrm{F}_{\mathrm{A}}\right)\right] \leq \operatorname{int}^{\mathrm{fs}}\left(\mathrm{H}_{\mathrm{c}}\right) \leq \operatorname{int}^{\mathrm{fs}}\left[1-\operatorname{int}^{\mathrm{fs}}\left(\mathrm{G}_{\mathrm{B}}\right)\right]$ and hence $\operatorname{int}^{\mathrm{fs}} \mathrm{cl}^{\mathrm{fs}}\left(\mathrm{F}_{\mathrm{A}}\right) \leq \mathrm{H}_{\mathrm{C}} \leq 1-\mathrm{cl}^{\mathrm{fs}}\left[\operatorname{int}^{\mathrm{fs}}\left(\mathrm{G}_{\mathrm{B}}\right)\right]$ in $(\mathrm{U}, \mathrm{E}, \psi)$. Then $\operatorname{int}^{\mathrm{fs}}\left\{1-\operatorname{cl}^{\mathrm{fs}}\left[\operatorname{int}^{\mathrm{fs}}\left(\mathrm{G}_{\mathrm{B}}\right)\right]\right\} \neq 0 . \Rightarrow 1-\operatorname{cl}^{\mathrm{fs}} \mathrm{cl}^{\mathrm{fs}}\left[\operatorname{int}^{\mathrm{fs}}\left(\mathrm{G}_{\mathrm{B}}\right)\right] \neq 0$ and then $\mathrm{cl}^{\mathrm{fs}}{ }^{\mathrm{int}}{ }^{\mathrm{fs}}\left(\mathrm{G}_{\mathrm{B}}\right) \neq 1$. Thus int ${ }^{\mathrm{fs}}\left(\mathrm{G}_{\mathrm{B}}\right)$ is not a fuzzy soft dense set in $(\mathrm{U}, \mathrm{E}, \psi)$.

\section{Proposition 3.5:}

If $F_{A} \leq 1-G_{B}$, where $G_{B}$ is a Fuzzy soft closed sets in Fuzzy soft pre dense sets $(\mathrm{U}, \mathrm{E}, \psi)$, then there exist a Fuzzy soft regular open set $\mathrm{H}_{\mathrm{c}}$ in $(\mathrm{U}, \mathrm{E}, \psi)$ such that int ${ }^{\mathrm{fs}}\left(\mathrm{F}_{\mathrm{A}}\right) \leq \mathrm{H}_{\mathrm{c}} \leq 1-\mathrm{G}_{\mathrm{B}}$.

\section{Proof:}

Suppose that $F_{A} \leq 1-G_{B}$, then we say $G_{B}$ is a Fuzzy soft closed sets in Fuzzy Soft Topological Spaces (U,E, $\psi$ ) . By prop 3.3, there exist a Fuzzy soft closed sets $\mathrm{L}_{\mathrm{D}}$ in FSTS . such that $\mathrm{F}_{\mathrm{A}} \leq$ $\mathrm{L}_{\mathrm{D}} \leq 1-\mathrm{G}_{\mathrm{B}} \Rightarrow \operatorname{int}^{\mathrm{fs}}\left(\mathrm{F}_{\mathrm{A}}\right) \leq \operatorname{int}^{\mathrm{fs}}\left(\mathrm{L}_{\mathrm{D}}\right) \leq \operatorname{int}^{\mathrm{fs}}\left(1-\mathrm{G}_{\mathrm{B}}\right)$, in FSTS $(\mathrm{U}, \mathrm{E}, \psi)$. Then , $\operatorname{int}^{\mathrm{fs}}\left(\mathrm{F}_{\mathrm{A}}\right) \leq \operatorname{int}^{\mathrm{fs}}\left(\mathrm{L}_{\mathrm{D}}\right) \leq \mathbf{1}-\mathrm{cl}^{\mathrm{fs}}\left(\mathrm{G}_{\mathrm{B}}\right)=1-\mathrm{G}_{\mathrm{B}}$, in $(\mathrm{U}, \mathrm{E}, \psi)$. Let $\mathrm{H}_{\mathrm{C}}=\mathrm{int}^{\mathrm{fs}}\left(\mathrm{L}_{\mathrm{D}}\right)$, the interior of a fuzzy soft regular open set in a FSTS [Thm2.1] $\mathrm{H}_{\mathrm{C}}$ is a fsrops in $(\mathrm{U}, \mathrm{E}, \psi)$. Hence there exist a Fuzzy soft regular open sets $\mathrm{H}_{\mathrm{c}}$ in $(\mathrm{U}, \mathrm{E}, \psi)$ such that int ${ }^{\mathrm{fs}}\left(\mathrm{F}_{\mathrm{A}}\right) \leq \mathrm{H}_{\mathrm{c}} \leq 1-\mathrm{G}_{\mathrm{B}}$.

\section{Proposition 3.6:}

If $F_{A} \leq 1-G_{B}$, where $F_{A}$ is a Fuzzy soft closed sets in Fuzzy soft pre dense sets $(\mathrm{U}, \mathrm{E}, \psi)$, then there exist a Fuzzy soft regular open sets $\mathrm{L}_{\mathrm{D}}$ in $(\mathrm{U}, \mathrm{E}, \psi)$ such that $\mathrm{F}_{\mathrm{A}} \leq \mathrm{L}_{\mathrm{D}} \leq 1-\operatorname{int}^{\mathrm{fs}}\left(\mathrm{G}_{\mathrm{B}}\right)$.

\section{Proof:}

Let $\mathrm{F}_{\mathrm{A}} \leq 1-\mathrm{G}_{\mathrm{B}}, \mathrm{F}_{\mathrm{A}}$ is a Fscs in $(\mathrm{U}, \mathrm{E}, \psi)$ then by prop 3.2, there exist a Fsops $\mathrm{H}_{\mathrm{C}}$ in $(\mathrm{U}, \mathrm{E}, \psi)$ such that $\mathrm{F}_{\mathrm{A}} \leq \mathrm{H}_{\mathrm{C}} \leq 1-\mathrm{G}_{\mathrm{B}} . \Rightarrow$ $\mathrm{cl}^{\mathrm{fs}}\left(\mathrm{F}_{\mathrm{A}}\right) \leq \mathrm{cl}^{\mathrm{fs}}\left(\mathrm{G}_{\mathrm{B}}\right) \leq \mathrm{cl}^{\mathrm{fs}}\left(1-\mathrm{G}_{\mathrm{B}}\right)$ in $(\mathrm{U}, \mathrm{E}, \psi)$. Then $\mathrm{F}_{\mathrm{A}} \leq \mathrm{cl}^{\mathrm{fs}}\left(\mathrm{H}_{\mathrm{c}}\right) \leq$ $1-\operatorname{int}^{\mathrm{fs}}\left(\mathrm{G}_{\mathrm{B}}\right)$, in $(\mathrm{U}, \mathrm{E}, \psi)$. Let $\mathrm{L}_{\mathrm{D}}=\mathrm{cl}^{\mathrm{fs}}\left(\mathrm{H}_{\mathrm{C}}\right)$ the closure of a Fuzzy soft open sets is a Fuzzy soft regular closed sets in $(\mathrm{U}, \mathrm{E}, \psi)$. Then there exist a regular closed set $\mathrm{L}_{\mathrm{D}}$ in $(\mathrm{U}, \mathrm{E}, \psi)$ such that $\mathrm{F}_{\mathrm{A}} \leq \mathrm{L}_{\mathrm{D}} \leq 1-\operatorname{int}^{\mathrm{fs}}\left(\mathrm{G}_{\mathrm{B}}\right)$.

\section{Proposition 3.7:}

If $F_{A} \leq 1-G_{B}$,for any two FSS $F_{A}$ and $G_{B}$ defined on $(U, E, \psi)$, then

(a) If $F_{A}$ is a fuzzy soft dense set in $(\mathrm{U}, \mathrm{E}, \psi)$, then $\mathrm{G}_{\mathrm{B}}$ is not a fuzzy soft open set in (U,E, $\psi)$.

(b) If $\mathrm{G}_{\mathrm{B}}$ is a fuzzy soft dense set in $(\mathrm{U}, \mathrm{E}, \psi)$, then $\mathrm{F}_{\mathrm{A}}$ is not a Fuzzy soft open sets in (U,E, $\psi)$.

(c) If $F_{A}$ is a fuzzy soft open sets in $(\mathrm{U}, \mathrm{E}, \psi)$, then $\mathrm{G}_{\mathrm{B}}$ is not a fuzzy soft dense set in (U,E, $\psi)$.

(d) If $\mathrm{G}_{\mathrm{B}}$ is a Fuzzy soft open sets in $(\mathrm{U}, \mathrm{E}, \psi)$, then $\mathrm{F}_{\mathrm{A}}$ is not a fuzzy soft dense set in $\quad(\mathrm{U}, \mathrm{E}, \psi)$.

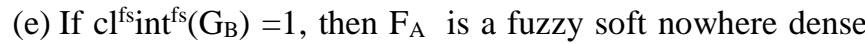
set in $(\mathrm{U}, \mathrm{E}, \psi)$.

\section{Proof:}

(i) Let $F_{A} \leq 1-G_{B}$, in $(U, E, \psi)$, then $\operatorname{cl}^{\mathrm{fs}}\left(F_{A}\right) \leq 1-$ int $^{f s}\left(G_{B}\right)$ in FSTS .Where $F_{A}$ is a fuzzy soft dense set in (U,E, $\psi$ ), $\mathrm{cl}^{\mathrm{fs}}\left(\mathrm{F}_{\mathrm{A}}\right)=1$ and $1 \leq 1-\operatorname{int}^{\mathrm{fs}}\left(\mathrm{G}_{\mathrm{B}}\right) . \Rightarrow \operatorname{int}^{\mathrm{fs}}\left(\mathrm{G}_{\mathrm{B}}\right)=0$ and Hence $\mathrm{G}_{\mathrm{B}}$ is not a fuzzy soft open set in $(\mathrm{U}, \mathrm{E}, \psi)$.

(ii) Now $F_{A} \leq 1-G_{B}$ in $(U, E, \psi)$ i.e $) \operatorname{cl}^{f^{f s}} \operatorname{int}^{\mathrm{fs}^{\mathrm{s}}}\left(\mathrm{F}_{\mathrm{A}}\right) \leq \mathrm{cl}^{\mathrm{fs}} \operatorname{int}^{\mathrm{fs}}\left(1-\mathrm{G}_{\mathrm{B}}\right)$. Then $\mathrm{cl}^{\mathrm{fs}} \operatorname{int}^{\mathrm{fs}}\left(\mathrm{F}_{\mathrm{A}}\right) \leq 1-$ int $^{\mathrm{fs}}\left[\mathrm{cl}^{\mathrm{fs}}\left(\mathrm{G}_{\mathrm{B}}\right)\right]$ in $(\mathrm{U}, \mathrm{E}, \psi)$. we say $\mathrm{G}_{\mathrm{B}}$ is a fuzzy soft dense set in $(\mathrm{U}, \mathrm{E}, \psi), \mathrm{cl}^{\mathrm{fs}}\left(\mathrm{G}_{\mathrm{B}}\right)=1$, Hence $\operatorname{int}^{\mathrm{fs}} \mathrm{cl}^{\mathrm{fs}}\left(\mathrm{G}_{\mathrm{B}}\right)=\operatorname{int}^{\mathrm{fs}}(1)=1$ in $(\mathrm{U}, \mathrm{E}, \psi)$. Then $\mathrm{cl}^{\mathrm{fs}} \operatorname{int}^{\mathrm{fs}}\left(\mathrm{F}_{\mathrm{A}}\right) \leq 1-\mathbf{1}$ $=0$ i.e $) \mathrm{cl}^{f_{s} \text { int }^{f s}}\left(\mathrm{~F}_{\mathrm{A}}\right)=0 . \Rightarrow \operatorname{int}^{\mathrm{fs}}\left(\mathrm{F}_{\mathrm{A}}\right)=0$, in $(\mathrm{U}, \mathrm{E}, \psi)$. Hence $\mathrm{F}_{\mathrm{A}}$ is not a fsops in $(\mathrm{U}, \mathrm{E}, \psi)$.

(iii) Let $\mathrm{F}_{\mathrm{A}} \leq 1-\mathrm{G}_{\mathrm{B}}$, in $(\mathrm{U}, \mathrm{E}, \psi)$, it shows that int ${ }^{\mathrm{fs}}\left(\mathrm{F}_{\mathrm{A}}\right) \leq \operatorname{int}^{\mathrm{fs}}(1-$ $\left.\mathrm{G}_{\mathrm{B}}\right)$ and $\mathrm{cl}^{\mathrm{fs}}\left(\mathrm{F}_{\mathrm{A}}\right) \leq 1-\mathrm{cl}^{\mathrm{fs}}\left(\mathrm{G}_{\mathrm{B}}\right)$ then $\mathrm{F}_{\mathrm{A}}$ is a fuzzy soft open 
sets int $^{\mathrm{fs}}\left(\mathrm{F}_{\mathrm{A}}\right)=\mathrm{F}_{\mathrm{A}}$ in FSTS $(\mathrm{U}, \mathrm{E}, \psi)$. Then $\mathrm{F}_{\mathrm{A}} \leq 1-\mathrm{cl}^{\mathrm{fs}}\left(\mathrm{F}_{\mathrm{A}}\right)$ $\Rightarrow \mathrm{cl}^{\mathrm{fs}}\left(\mathrm{G}_{\mathrm{B}}\right) \leq 1-\mathrm{F}_{\mathrm{A}}$ and hence $\mathrm{cl}^{\mathrm{fs}}\left(\mathrm{G}_{\mathrm{B}}\right) \neq 1$ in $(\mathrm{U}, \mathrm{E}, \psi)$, therefore $\mathrm{G}_{\mathrm{B}}$ is not a fuzzy soft dense set in $(\mathrm{U}, \mathrm{E}, \psi)$.

(iv) since $\mathrm{F}_{\mathrm{A}} \leq 1-\mathrm{G}_{\mathrm{B}}$, where $\mathrm{cl}^{\mathrm{fs}}\left(\mathrm{F}_{\mathrm{A}}\right) \leq \mathrm{cl}^{\mathrm{fs}}\left(1-\mathrm{G}_{\mathrm{B}}\right)$ and hence $\mathrm{cl}^{\mathrm{fs}}\left(\mathrm{F}_{\mathrm{A}}\right) \leq 1-\operatorname{int}^{\mathrm{fs}}\left(\mathrm{G}_{\mathrm{B}}\right)$ then $\mathrm{G}_{\mathrm{B}}$ is a fuzzy soft openset in $(\mathrm{U}, \mathrm{E}, \psi)$, int ${ }^{\mathrm{fs}}\left(\mathrm{G}_{\mathrm{B}}\right)=\mathrm{G}_{\mathrm{B}}$ and thus $\mathrm{cl}^{\mathrm{fs}}\left(\mathrm{F}_{\mathrm{A}}\right) \leq 1-\mathrm{G}_{\mathrm{B}}$. Hence $\mathrm{cl}^{\mathrm{fs}}\left(\mathrm{F}_{\mathrm{A}}\right) \neq 1$, in $(\mathrm{U}, \mathrm{E}, \psi)$.

$\therefore \mathrm{F}_{\mathrm{A}}$ is not a fuzzy soft dense set in (U,E, $\left.\psi\right)$.

(v) Let $F_{A} \leq 1-G_{B} \Rightarrow \operatorname{int}^{f s} \operatorname{cl}^{\mathrm{fs}}\left(F_{A}\right) \leq$ int $^{f s} \operatorname{cl}^{\mathrm{fs}}\left(1-G_{B}\right)$ and then int ${ }^{\mathrm{fs}}$ $\mathrm{cl}^{\mathrm{fs}}\left(\mathrm{F}_{\mathrm{A}}\right) \leq 1-\mathrm{cl}^{\mathrm{fs}} \operatorname{int}^{\mathrm{fs}}\left(\mathrm{G}_{\mathrm{B}}\right)$ in $(\mathrm{U}, \mathrm{E}, \psi)$. since $\mathrm{cl}^{\mathrm{fs}} \operatorname{int}^{\mathrm{fs}}\left(\mathrm{G}_{\mathrm{B}}\right)=1$, int $^{\mathrm{fs}} \mathrm{cl}^{\mathrm{fs}}\left(\mathrm{F}_{\mathrm{A}}\right) 1-1=0$ (I.e) int $^{\mathrm{fs}} \mathrm{cl}^{\mathrm{fs}}\left(\mathrm{F}_{\mathrm{A}}\right)=0$ in $(\mathrm{U}, \mathrm{E}, \psi)$.

$\therefore \mathrm{F}_{\mathrm{A}}$ is a fuzzy soft nowhere dense set in (U,E, $\psi$ ).

\section{Proposition 3.8:}

If $F_{A}$ is a FSS in Fuzzy soft pre dense sets $(U, E, \psi)$ then $\operatorname{int}^{f \mathrm{fs}}\left(\mathrm{F}_{\mathrm{A}}\right)$ is a Fuzzy soft closed sets in (U,E, $\psi)$.

\section{Proof:}

Let $F_{A}$ be a Fss defined on $U$ the fuzzy soft set $1-F_{A}, 1-F_{A} \leq$ $\mathrm{cl}^{\mathrm{fs}}\left(1-\mathrm{F}_{\mathrm{A}}\right)$.then $1-\mathrm{F}_{\mathrm{A}} \leq \mathrm{cl}^{\mathrm{fs}}\left(1-\mathrm{F}_{\mathrm{A}}\right)$. Then $\left(1-\mathrm{F}_{\mathrm{A}}\right) \leq 1-$ int $^{\mathrm{fs}}\left(\mathrm{F}_{\mathrm{A}}\right)$. since $\operatorname{int}^{\mathrm{fs}}\left(\mathrm{F}_{\mathrm{A}}\right)$ is a Fuzzy soft open sets in $(\mathrm{U}, \mathrm{E}, \psi)$ by prop $3.7,1-\mathrm{F}_{\mathrm{A}}$ is not a fuzzy soft dense set in $(\mathrm{U}, \mathrm{E}, \psi)$. ie) $\mathrm{cl}^{\mathrm{fs}}\left(1-\mathrm{F}_{\mathrm{A}}\right) \neq 1$ in $(\mathrm{U}, \mathrm{E}, \psi)$ and $\operatorname{int}^{\mathrm{fs}}\left(\mathrm{F}_{\mathrm{A}}\right) \neq 0$ in $(\mathrm{U}, \mathrm{E}, \psi)$. since $(\mathrm{U}, \mathrm{E}, \psi)$ is a Fuzzy soft pre dense sets $\left(1-\mathrm{F}_{\mathrm{A}}\right) \leq 1-\operatorname{int}^{\mathrm{fs}}\left(\mathrm{F}_{\mathrm{A}}\right)$ in $(\mathrm{U}, \mathrm{E}, \psi), \mathrm{cl}^{\mathrm{fs}}\left(1-\mathrm{F}_{\mathrm{A}}\right) \leq 1-$ $\mathrm{cl}^{\mathrm{fs}}\left[\operatorname{int}^{\mathrm{fs}}\left(\mathrm{F}_{\mathrm{A}}\right)\right]$ and then $1-\operatorname{int}^{\mathrm{fs}}\left(\mathrm{F}_{\mathrm{A}}\right) \leq 1-\mathrm{cl}^{\mathrm{fs}}\left[\operatorname{int}^{\mathrm{fs}}\left(\mathrm{F}_{\mathrm{A}}\right)\right]$ and hence $\operatorname{cl}^{\mathrm{fs}}\left[\operatorname{int}^{\mathrm{fs}}\left(\mathrm{F}_{\mathrm{A}}\right)\right] \leq \operatorname{int}^{\mathrm{fs}}\left(\mathrm{F}_{\mathrm{A}}\right)$. But int ${ }^{\mathrm{fs}}\left(\mathrm{F}_{\mathrm{A}}\right) \leq \mathrm{cl}^{\mathrm{fs}}\left[\operatorname{int}^{\mathrm{fs}}\left(\mathrm{F}_{\mathrm{A}}\right)\right]$ in $(\mathrm{U}, \mathrm{E}, \psi)$. This implies that $\operatorname{cl}^{\mathrm{fs}}\left[\operatorname{int}^{\mathrm{fs}}\left(\mathrm{F}_{\mathrm{A}}\right)\right]=\operatorname{int}^{\mathrm{fs}}\left(\mathrm{F}_{\mathrm{A}}\right)$ therefore $\operatorname{int}^{\mathrm{fs}}\left(\mathrm{F}_{\mathrm{A}}\right)$ is a Fscs in (U,E, $\psi)$.

\section{Proposition 3.9:}

IF $F_{A}$ is a Fuzzy Soft Sets in Fuzzy Soft pre dense sets (U,E, $\psi$ ) ,then $\mathrm{F}_{\mathrm{A}}$ is a fuzzy soft pre -closed set in (U,E, $\left.\psi\right)$.

\section{Proof:}

Let $F_{A}$ be a Fuzzy Soft sets defined on $U$ in a Fuzzy Soft Pre dense Sets $(\mathrm{U}, \mathrm{E}, \psi)$. For prop 3.8, int $^{\mathrm{fs}}\left(\mathrm{F}_{\mathrm{A}}\right)$ is a Fuzzy soft closed sets in $(\mathrm{U}, \mathrm{E}, \psi)$.Let $\mathrm{G}_{\mathrm{B}}=\operatorname{int}^{\mathrm{fs}}\left(\mathrm{F}_{\mathrm{A}}\right)$. since $\mathrm{G}_{\mathrm{B}}$ is a Fuzzy soft closed sets, $\mathrm{cl}^{\mathrm{fs}}\left(\mathrm{G}_{\mathrm{B}}\right)=\mathrm{G}_{\mathrm{B}}$, in $(\mathrm{U}, \mathrm{E}, \psi)$. Then $\mathrm{cl}^{\mathrm{fs}}$ int $^{\mathrm{fs}}\left(\mathrm{F}_{\mathrm{A}}\right)=$ $\operatorname{int}^{\mathrm{fs}}\left(\mathrm{F}_{\mathrm{A}}\right)$ and then $\mathrm{cl}^{\mathrm{fs}} \operatorname{int}^{\mathrm{fs}}\left(\mathrm{F}_{\mathrm{A}}\right)=\operatorname{int}^{\mathrm{fs}}\left(\mathrm{F}_{\mathrm{A}}\right) \leq \mathrm{F}_{\mathrm{A}}$ in $(\mathrm{U}, \mathrm{E}, \psi)$. since cl $^{\mathrm{fs}}$ int $^{\mathrm{fs}}\left(\mathrm{F}_{\mathrm{A}}\right) \leq \mathrm{F}_{\mathrm{A}}$, this implies that $\mathrm{F}_{\mathrm{A}}$ is a fuzzy soft pre-closed set in $(\mathrm{U}, \mathrm{E}, \psi)$.

\section{Remark 3.1:}

In view of the above proposition following results "IF $F_{A}$ is a Fuzzy Soft Sets in a Fuzzy soft pre dense sets $(\mathrm{U}, \mathrm{E}, \psi)$, then 1$\mathrm{F}_{\mathrm{A}}$ is a fuzzy soft pre -open set in $(\mathrm{U}, \mathrm{E}, \psi)$.

\section{Remark 3.2}

In a FSTS $(\mathrm{U}, \mathrm{E}, \varphi)$ the non-zero Fuzzy soft open sets $\left(F_{A_{i}}\right)$ 's are not Fuzzy soft nowhere dense sets , $F_{A}=\operatorname{int}^{f \mathrm{fs}}\left(\mathrm{F}_{\mathrm{A}}\right) \leq \operatorname{int}^{\mathrm{fs}} \mathrm{cl}^{\mathrm{fs}}\left(\mathrm{F}_{\mathrm{A}}\right)$ and thus int ${ }^{\mathrm{fs}} \mathrm{Cl}^{\mathrm{fs}}\left(\mathrm{F}_{\mathrm{A}}\right) \neq 0$ in $(\mathrm{U}, \mathrm{E}, \psi)$

\section{Proposition3.10}

If $F_{A}$ is a Fuzzy soft open sets in Fuzzy soft pre dense sets $(\mathrm{U}, \mathrm{E}, \psi)$, then $\mathrm{F}_{\mathrm{A}}$ is not a fuzzy soft nowhere dense set in $(\mathrm{U}, \mathrm{E}, \psi)$.

\section{Proof:}

The proof follows from remark 3.2

\section{Proposition3.11:}

If $\mathrm{F}_{\mathrm{A}}$ is a fuzzy soft regular open set in Fuzzy soft pre dense sets $(\mathrm{U}, \mathrm{E}, \psi)$, then $\mathrm{F}_{\mathrm{A}}$ is not a fuzzy soft nowhere dense set in $(\mathrm{U}, \mathrm{E}, \psi)$.

\section{Proof:}

The proof follows from remark 3.2.

\section{Proposition 3.12:}

If $F_{A}$ is not a fuzzy soft nowhere dense set in a Fuzzy Soft Pre dense $(\mathrm{U}, \mathrm{E}, \psi)$, then $\operatorname{int}^{\mathrm{fs}}\left[\mathrm{cl}^{\mathrm{fs}}\left(\mathrm{F}_{\mathrm{A}}\right)\right]$ is a Fuzzy Soft Closed Sets in $(\mathrm{U}, \mathrm{E}, \psi)$.

\section{Proof :}

Let $\mathrm{F}_{\mathrm{A}}$ is not a Fuzzy soft nowhere dense sets in $(\mathrm{U}, \mathrm{E}, \psi)$, then $\operatorname{int}^{\mathrm{fs}} \mathrm{cl}^{\mathrm{fs}}\left(\mathrm{F}_{\mathrm{A}}\right) \neq 0$ in $(\mathrm{U}, \mathrm{E}, \psi)$. Now $\operatorname{int}^{\mathrm{fs}} \mathrm{cl}^{\mathrm{fs}}\left(\mathrm{F}_{\mathrm{A}}\right) \leq \mathrm{cl}^{\mathrm{fs}}\left(\mathrm{F}_{\mathrm{A}}\right)$ in $(\mathrm{U}, \mathrm{E}, \psi)$ and then $\operatorname{int}^{\mathrm{fs}} \mathrm{cl}^{\mathrm{fs}}\left(\mathrm{F}_{\mathrm{A}}\right) \leq 1-\left[1-\mathrm{cl}^{\mathrm{fs}}\left(\mathrm{F}_{\mathrm{A}}\right)\right]$ in $(\mathrm{U}, \mathrm{E}, \psi)$, its fuzzy soft perfectly disconnectedness of $(\mathrm{U}, \mathrm{E}, \psi)$. We have $\mathrm{cl}^{\mathrm{fs}}\left[\operatorname{int}^{\mathrm{fs}} \mathrm{cl}^{\mathrm{fs}}\left(\mathrm{F}_{\mathrm{A}}\right)\right] \leq 1-\mathrm{cl}^{\mathrm{fs}}\left[1-\mathrm{cl}^{\mathrm{fs}}\left(\mathrm{F}_{\mathrm{A}}\right)\right]$ and then $\mathrm{cl}^{\mathrm{fs}}\left[\operatorname{int}^{\mathrm{fs}} \mathrm{cl}^{\mathrm{fs}}\left(\mathrm{F}_{\mathrm{A}}\right)\right] \leq 1-$ $\left[1-\operatorname{int}^{\mathrm{fs}} \mathrm{cl}^{\mathrm{fs}}\left(\mathrm{F}_{\mathrm{A}}\right)\right]$ in $(\mathrm{U}, \mathrm{E}, \psi)$. This implies $\mathrm{cl}^{\mathrm{fs}}\left[\operatorname{int}^{\mathrm{fs}} \mathrm{cl}^{\mathrm{fs}}\left(\mathrm{F}_{\mathrm{A}}\right)\right] \leq$ $\operatorname{int}^{\mathrm{fs}}\left[\mathrm{cl}^{\mathrm{fs}}\left(\mathrm{F}_{\mathrm{A}}\right)\right]$. But int ${ }^{\mathrm{fs}}\left[\mathrm{cl}^{\mathrm{fs}}\left(\mathrm{F}_{\mathrm{A}}\right)\right] \leq \mathrm{cl}^{\mathrm{fs}}\left[\operatorname{int}^{\mathrm{fs}} \mathrm{cl}^{\mathrm{fs}}\left(\mathrm{F}_{\mathrm{A}}\right)\right]$ in $(\mathrm{U}, \mathrm{E}, \psi)$ thus $\mathrm{cl}^{\mathrm{fs}}\left[\operatorname{int}^{\mathrm{fs}} \mathrm{cl}^{\mathrm{fs}}\left(\mathrm{F}_{\mathrm{A}}\right)\right]=\operatorname{int}^{\mathrm{fs}} \mathrm{cl}^{\mathrm{fs}}\left(\mathrm{F}_{\mathrm{A}}\right)$. Then, $\operatorname{int}^{\mathrm{fs}} \mathrm{cl}^{\mathrm{fs}}\left(\mathrm{F}_{\mathrm{A}}\right)$. Then int $^{\mathrm{fs}} \mathrm{Cl}^{\mathrm{fs}}\left(\mathrm{F}_{\mathrm{A}}\right)$ is a Fuzzy soft closed sets in $(\mathrm{U}, \mathrm{E}, \psi)$.

\section{Proposition 3.13:}

If $F_{A}$ is a Fuzzy soft regular open sets in a Fuzzy soft pre dense sets $(\mathrm{U}, \mathrm{E}, \psi)$, then $\operatorname{int}^{\mathrm{fs}} \mathrm{cl}^{\mathrm{fs}}\left(\mathrm{F}_{\mathrm{A}}\right)$ is a Fuzzy soft closed sets in $(\mathrm{U}, \mathrm{E}, \psi)$.

\section{Proof:}

Let $\mathrm{F}_{\mathrm{A}}$ be a non-zero Fuzzy soft open sets in $(\mathrm{U}, \mathrm{E}, \psi)$ is a Fuzzy soft pre dense sets ,by propo $3.10, \mathrm{~F}_{\mathrm{A}}$ is not a fuzzy soft nowhere dense set in $(\mathrm{U}, \mathrm{E}, \psi)$. Then ,by prop 3.12, int ${ }^{\mathrm{fs}} \mathrm{cl}^{\mathrm{fs}}\left(\mathrm{F}_{\mathrm{A}}\right)$ is a Fuzzy soft closed sets in $(\mathrm{U}, \mathrm{E}, \psi)$.

\section{Proposition 3.14}

If $\mathrm{F}_{\mathrm{A}}$ is a Fuzzy soft regular open sets in a Fuzzy soft pre dense sets $(\mathrm{U}, \mathrm{E}, \psi)$, then $\mathrm{F}_{\mathrm{A}}$ is a Fuzzy soft closed sets in $(\mathrm{U}, \mathrm{E}, \psi)$.

\section{Proof:}

Let $\mathrm{F}_{\mathrm{A}}$ be a Fuzzy soft regular open sets in $(\mathrm{U}, \mathrm{E}, \psi)$ is a Fuzzy soft pre dense sets ,by prop 3.11, $\mathrm{F}_{\mathrm{A}}$ is not a Fuzzy soft nowhere 
dense sets in $(\mathrm{U}, \mathrm{E}, \psi)$ and hence by prop 3.13, $\operatorname{int}^{\mathrm{fs}} \mathrm{cl}^{\mathrm{fs}}\left(\mathrm{F}_{\mathrm{A}}\right)$ is a Fuzzy soft closed sets in $(\mathrm{U}, \mathrm{E}, \psi), \mathrm{F}_{\mathrm{A}}$ is a fuzzy soft regular open sets in $(\mathrm{U}, \mathrm{E}, \psi)$, int ${ }^{\mathrm{fs}} \mathrm{cl}^{\mathrm{fs}}\left(\mathrm{F}_{\mathrm{A}}\right)=\mathrm{F}_{\mathrm{A}}$ and $\mathrm{F}_{\mathrm{A}}$ is a Fuzzy soft closed sets in (U,E, $\psi)$.

\section{Proposition 3.15:}

If $\mathrm{F}_{\mathrm{A}}$ is a Fuzzy soft regular closed sets in a Fuzzy soft pre dense sets $(\mathrm{U}, \mathrm{E}, \psi)$, then $\mathrm{F}_{\mathrm{A}}$ is a Fuzzy soft open sets in $(\mathrm{U}, \mathrm{E}, \psi)$.

\section{Proof:}

Let $\mathrm{F}_{\mathrm{A}}$ be a Fuzzy soft regular closed sets in $(\mathrm{U}, \mathrm{E}, \psi), 1-\mathrm{F}_{\mathrm{A}}$ is a Fuzzy soft regular open sets in $(\mathrm{U}, \mathrm{E}, \psi)$ is a Fuzzy soft pre dense sets, by prop 3.14, 1-F is a Fuzzy soft closed sets in $(\mathrm{U}, \mathrm{E}, \psi) . \mathrm{F}_{\mathrm{A}}$ is a Fuzzy soft open sets in $(\mathrm{U}, \mathrm{E}, \psi)$.

\section{Proposition 3.16:}

If $\mathrm{F}_{\mathrm{A}}=\left[\mathrm{V}_{i=1}^{\infty}\left(F_{A_{i}}\right)\right]$, where $\left(F_{A_{i}}\right)$ are Fuzzy soft sets defined on a Fspds (U,E, $\psi)$, then $\mathrm{F}_{\mathrm{A}}$ is a fuzzy soft pre $F_{\sigma}-$ set in $(\mathrm{U}, \mathrm{E}, \psi)$.

\section{Proof:}

Let $\mathrm{F}_{\mathrm{A}}=\left[\mathrm{V}_{i=1}^{\infty}\left(F_{A_{i}}\right)\right]$. where $\left(F_{A_{i}}\right)$ 's are fuzzy soft sets defined on the Fspds (U,E, $\psi)$. Then prop 3.9, $\left(F_{A_{i}}\right)$ 's are fuzzy soft pre -closed sets in (U,E, $\psi$ ) and hence $\mathrm{F}_{\mathrm{A}}$ is a fuzzy soft pre $F_{\sigma}-$ set in $(\mathrm{U}, \mathrm{E}, \psi)$.

\section{Proposition 3.17:}

If $(\mathrm{U}, \mathrm{E}, \psi)$ is a Fuzzy soft pre dense sets ,then

(i) $\quad \mathrm{Cl}^{\mathrm{fs}}{ }^{\mathrm{int}}{ }^{\mathrm{fs}}\left(\mathrm{F}_{\mathrm{A}}\right)+\mathrm{cl}^{\mathrm{fs}}\left(1-\mathrm{F}_{\mathrm{A}}\right) \leq 1$, for a Fss $\mathrm{F}_{\mathrm{A}}$ defined on $\mathrm{U}$.

(ii) For any two Fss $F_{A}$ and $G_{B}$, with $F_{A}+G_{B} \leq 1$, clfs $^{f^{f s}}\left(F_{A}\right)$ $+\mathrm{cl}^{\mathrm{fs}}\left(\mathrm{G}_{\mathrm{B}}\right) \leq 1$.

(iii) For any two Fss $F_{A}$ and $G_{B}$ with $F_{A}+G_{B} \leq 1$, cl $^{\mathrm{fs}_{\text {int }}}{ }^{\mathrm{fs}}\left(\mathrm{F}_{\mathrm{A}}\right)$ $+\operatorname{cl}^{\mathrm{fs}} \operatorname{int}^{\mathrm{fs}}\left(\mathrm{G}_{\mathrm{B}}\right) \leq 1$.

\section{Proof:}

Let $F_{A}$ be a Fuzzy soft sets defined on $U$,int ${ }^{\text {fs }}\left(F_{A}\right) \leq F_{A}$ and thus

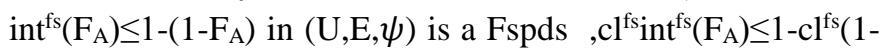
$\left.\mathrm{F}_{\mathrm{A}}\right)$, in $(\mathrm{U}, \mathrm{E}, \psi)$ and hence $\mathrm{Cl}^{\mathrm{fs}} \operatorname{int}^{\mathrm{fs}}\left(\mathrm{F}_{\mathrm{A}}\right)+\mathrm{cl}^{\mathrm{fs}}\left(1-\mathrm{F}_{\mathrm{A}}\right) \leq 1$.

(ii) Suppose that $F_{A}+G_{B} \leq 1$, for any two Fss $F_{A}$ and $G_{B}$ defined on $\mathrm{U}$. Then $\mathrm{F}_{\mathrm{A}} \leq 1-\mathrm{G}_{\mathrm{B}}$, in $(\mathrm{U}, \mathrm{E}, \psi)$ is a Fspds, $\mathrm{cl}^{\mathrm{fs}}\left(\mathrm{F}_{\mathrm{A}}\right) \leq \mathrm{cl}^{\mathrm{fs}}(1-$ $\left.\mathrm{G}_{\mathrm{B}}\right)$, in $(\mathrm{U}, \mathrm{E}, \psi)$. Then $\operatorname{int}^{\mathrm{fs}}\left(\mathrm{F}_{\mathrm{A}}\right) \leq 1-$-int $^{\mathrm{fs}}\left(\mathrm{G}_{\mathrm{B}}\right)$ in $(\mathrm{U}, \mathrm{E}, \psi)$, Hence $\mathrm{cl}^{\mathrm{fs}}\left(\mathrm{F}_{\mathrm{A}}\right)+\mathrm{cl}^{\mathrm{fs}}\left(\mathrm{G}_{\mathrm{B}}\right) \leq 1$.

(iii)suppose that $F_{A}+G_{B} \leq 1, F_{A}$ and $G_{B}$ are two subsets its defined on $\mathrm{U}$. Then $\mathrm{F}_{\mathrm{A}} \leq 1-\mathrm{G}_{\mathrm{B}}$, in $(\mathrm{U}, \mathrm{E}, \psi)$, then $\operatorname{int}^{\mathrm{fs}}\left(\mathrm{F}_{\mathrm{A}}\right) \leq 1$ $\operatorname{int}^{\mathrm{fs}}\left(\mathrm{G}_{B}\right)$ in $(\mathrm{U}, \mathrm{E}, \psi)$ is a Fuzzy soft pre dense sets, $\mathrm{cl}^{\mathrm{fs}}$ int $^{\mathrm{fs}}\left(\mathrm{F}_{\mathrm{A}}\right) \leq 1-\mathrm{cl}^{\mathrm{fs}} \operatorname{int}^{\mathrm{fs}}\left(\mathrm{G}_{\mathrm{B}}\right)$ in $(\mathrm{U}, \mathrm{E}, \psi)$ and hence

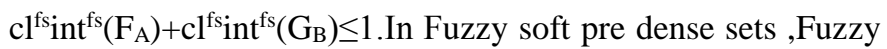
soft open sets are not Fuzzy soft dense sets .

\section{Proposition 3.18:}

If $(\lambda \neq 1)$ is a Fuzzy soft open sets in a Fuzzy soft pre dense sets $(\mathrm{U}, \mathrm{E}, \psi)$, then $\mathrm{F}_{\mathrm{A}}$ is not a Fuzzy soft pre dense sets in $(\mathrm{U}, \mathrm{E}, \psi)$.

\section{Proof:}

Let $\mathrm{F}_{\mathrm{A}}$ be a Fsops (U,E, $\psi$ ) .Now by prop 3.17, $\mathrm{cl}^{\mathrm{fs}} \operatorname{int}^{\mathrm{fs}}\left(\mathrm{F}_{\mathrm{A}}\right)+\mathrm{cl}^{\mathrm{fs}}\left(1-\mathrm{F}_{\mathrm{A}}\right) \leq 1$ in $(\mathrm{U}, \mathrm{E}, \psi)$. Then we say

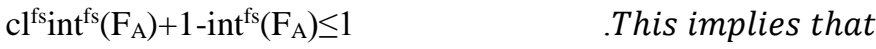
$\operatorname{cl}^{\mathrm{fs}} \operatorname{int}^{\mathrm{fs}}\left(\mathrm{F}_{\mathrm{A}}\right) \leq \operatorname{int}^{\mathrm{fs}}\left(\mathrm{F}_{\mathrm{A}}\right)$. But $\quad \operatorname{int}^{\mathrm{fs}}\left(\mathrm{F}_{\mathrm{A}}\right) \leq \quad \operatorname{cl}^{\mathrm{fs}} \operatorname{int}^{\mathrm{fs}}\left(\mathrm{F}_{\mathrm{A}}\right) \quad$ Hence $\operatorname{cl}^{\mathrm{fs}}\left[\operatorname{int}^{\mathrm{fs}}\left(\mathrm{F}_{\mathrm{A}}\right)\right]=\operatorname{int}^{\mathrm{fs}}\left(\mathrm{F}_{\mathrm{A}}\right) \cdot \mathrm{F}_{\mathrm{A}}$ is a Fuzzy soft open sets ,int ${ }^{\mathrm{fs}}\left(\mathrm{F}_{\mathrm{A}}\right)$ $=\mathrm{F}_{\mathrm{A}}$ and then $\mathrm{cl}^{\mathrm{fs}}\left(\mathrm{F}_{\mathrm{A}}\right)=\mathrm{F}_{\mathrm{A}} \neq 1, \mathrm{~F}_{\mathrm{A}}$ is not a Fuzzy soft dense sets in $(\mathrm{U}, \mathrm{E}, \psi)$.

\section{FUZZY SOFT DISCONNECTED SPACES ON FUZZY SOFT TOPOLOGICAL SPACES Proposition 4.1:}

If $(\mathrm{U}, \mathrm{E}, \psi)$ is a Fuzzy soft pre dense sets ,then $(\mathrm{U}, \mathrm{E}, \psi)$ is a fuzzy soft extremally disconnected space.

\section{Proof:}

Suppose that $\mathrm{F}_{\mathrm{A}}$ be a Fss defined on $\mathrm{U}$. (U,E, $\left.\psi\right)$ is a Fspds and $\left(1-\mathrm{F}_{\mathrm{A}}\right) \leq 1-\left[\operatorname{int}^{\mathrm{fs}}\left(\mathrm{F}_{\mathrm{A}}\right)\right]$ in $(\mathrm{U}, \mathrm{E}, \psi) \Rightarrow \mathrm{cl}^{\mathrm{fs}}\left(1-\mathrm{F}_{\mathrm{A}}\right) \leq 1-\mathrm{cl}^{\mathrm{fs}}\left[\operatorname{int}^{\mathrm{fs}}\left(\mathrm{F}_{\mathrm{A}}\right)\right]$

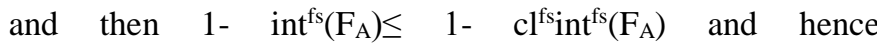
$\operatorname{cl}^{\mathrm{fs}} \operatorname{int}^{\mathrm{fs}}\left(\mathrm{F}_{\mathrm{A}}\right) \leq \operatorname{int}^{\mathrm{fs}}\left(\mathrm{F}_{\mathrm{A}}\right)$. But $\quad \operatorname{int}^{\mathrm{fs}}\left(\mathrm{F}_{\mathrm{A}}\right) \leq \quad \operatorname{cl}^{\mathrm{fs}}\left[\operatorname{int}^{\mathrm{fs}}\left(\mathrm{F}_{\mathrm{A}}\right)\right] \quad$ in $(\mathrm{U}, \mathrm{E}, \psi) \cdot \mathrm{cl}^{\mathrm{fs}} \operatorname{int}^{\mathrm{fs}}\left(\mathrm{F}_{\mathrm{A}}\right)=\operatorname{int}^{\mathrm{fs}}\left(\mathrm{F}_{\mathrm{A}}\right)$, in $(\mathrm{U}, \mathrm{E}, \psi)$. Let $\mathrm{L}_{\mathrm{D}}=\operatorname{int}^{\mathrm{fs}}\left(\mathrm{F}_{\mathrm{A}}\right)$, then $\mathrm{G}_{\mathrm{B}}$ is a Fuzzy soft open sets in $(\mathrm{U}, \mathrm{E}, \psi)$. Now $\mathrm{cl}^{\mathrm{fs}}\left(\mathrm{G}_{\mathrm{B}}\right)=\mathrm{L}_{\mathrm{D}}$ and $\mathrm{G}_{\mathrm{B}} \in \mathrm{T}$ this implies that $\mathrm{cl}^{\mathrm{fs}}\left(\mathrm{G}_{\mathrm{B}}\right) \in T$ if $G_{B} \in$ $T$, then $\mathrm{cl}^{f s}\left(G_{B}\right) \in T$. (U,E, $\left.\psi\right)$ is a Fuzzy soft extremally disconnected spaces.

\section{Proposition 4.2:}

If $(\mathrm{U}, \mathrm{E}, \psi)$ is a Fuzzy soft pre dense sets, then $(\mathrm{U}, \mathrm{E}, \psi)$ is not a fuzzy soft weakly Baire space.

\section{Proof:}

Let $(\mathrm{U}, \mathrm{E}, \psi)$ be a Fspds and $\left(F_{A_{i}}\right)$ 's are (i=1 to $\left.\infty\right)$ be non-zero regular open sets in $(\mathrm{U}, \mathrm{E}, \psi)$. Let $G_{B_{i}}=c l^{f s}\left(F_{A_{i}}\right) \wedge(1-$ $\left.F_{A_{i}}\right)$. Then int ${ }^{f s}\left(G_{B_{i}}\right)=\operatorname{int}^{f s}\left[c l^{f s}\left(F_{A_{i}}\right) \wedge\left(1-F_{A_{i}}\right)\right]=$ int $^{f s}\left[c l^{f s}\left(F_{A_{i}}\right)\right] \wedge \operatorname{int}^{f s}\left[\left(1-F_{A_{i}}\right)\right]$. since the $\operatorname{FSS}\left(F_{A_{i}}\right)$ 's are fuzzy soft regular open sets in $(\mathrm{U}, \mathrm{E}, \psi) \cdot \operatorname{int}^{f s}\left[\mathrm{cl}^{\mathrm{fs}}\left(F_{A_{i}}\right)\right]=$ $F_{A_{i}}$, and thus int ${ }^{f s}\left(G_{B_{i}}\right)=\left(F_{A_{i}}\right) \wedge i n t^{f s}[(1-$ $\left.F_{A_{i}}\right)$ ]. By prop 3.14, $\left(F_{A_{i}}\right)^{\prime} S$ are FSCS in $(U, E, \psi)$. By prop 3.8, $\left[i n t^{f s}\left(1-F_{A_{i}}\right)\right]^{\prime} s$ are Fscs in $(U, E, \psi)$. Then $\left\{F_{A_{i}} \Lambda \operatorname{int}^{\mathrm{fs}}((1-\right.$ $\left.\left.F_{A_{i}}\right)\right\}^{\prime}$ s are FSCS in (U,E, $\left.\psi\right)$ and thus $\left[\operatorname{int}^{\mathrm{fs}}\left(G_{B_{i}}\right)\right.$ ]'s are nonzero Fuzzy Soft Closed Sets in $(\mathrm{U}, \mathrm{E}, \psi), \Rightarrow \mathrm{V}_{i=1}^{\infty} \operatorname{int}\left(G_{B_{i}}\right) \neq$ 0 . By lemma 2.2, $\bigvee_{\infty}^{i=1} \operatorname{int}\left(G_{B_{i}}\right) \leq i n t^{f s}\left[\bigvee_{i=1}^{\infty}\left(G_{B_{i}}\right)\right] \neq$ 0 , where $G_{B_{i}}=\left(F_{A_{i}}\right) \wedge(1-$ $\left.F_{A_{i}}\right)$ and $\left(F_{A_{i}}\right)^{\prime}$ s are Fsrops in (U,E, $\left.\psi\right)$, is not a fuzzy soft weakly baire space. 


\section{Proposition 4.3:}

If $\operatorname{Pint}^{\mathrm{fs}}\left[\mathrm{V}_{i=1}^{\infty}\left(F_{A_{i}}\right)\right]=0$, where $\operatorname{pint}\left(F_{A_{i}}\right)=0$, for the Fuzzy Soft Sets $\left(F_{A_{i}}\right)^{\prime}$ s are Fspds in $(\mathrm{U}, \mathrm{E}, \psi)$, then $(\mathrm{U}, \mathrm{E}, \psi)$ is a fuzzy soft pre -Baire space.

Proof: Let $\left(F_{A_{i}}\right)^{\prime} s(i=1$ to $\infty)$ be FSS defined on a Fspds $(\mathrm{U}, \mathrm{E}, \psi)$. Then prop 3.9, and $\left(F_{A_{i}}\right)^{\prime}$ s are Fuzzy soft pre closed sets in $(\mathrm{U}, \mathrm{E}, \psi)$. Now $\operatorname{pint}^{\mathrm{fs}}\left[\operatorname{pcl}^{\mathrm{fs}}\left(F_{A_{i}}\right)\right]=\operatorname{pint}^{\mathrm{fs}}\left(F_{A_{i}}\right)=$ 0 (by hypothesis ), hence $\left(F_{A_{i}}\right)^{\prime} s$ are fuzzy soft pre-nowhere dense sets in $(\mathrm{U}, \mathrm{E}, \psi)$, Hence $\operatorname{Pint}^{\mathrm{fs}}\left[\mathrm{V}_{i=1}^{\infty}\left(F_{A_{i}}\right)\right]=$ $0,\left(F_{A_{i}}\right)^{\prime} s$ are fuzzy soft pre nowhere dense sets in, (U, $\mathrm{E}, \psi)$ this implies that $(\mathrm{U}, \mathrm{E}, \psi)$ is a fuzzy soft pre-Baire space.

\section{Proposition 4.4:}

If $(\mathrm{U}, \mathrm{E}, \psi)$ is a Fuzzy Soft pre dense sets ,then $(\mathrm{U}, \mathrm{E}, \psi)$ is not a fuzzy soft hyper connected space.

\section{Proof:}

Let $\mathrm{F}_{\mathrm{A}}$ be a fuzzy soft open sets in $(\mathrm{U}, \mathrm{E}, \psi)$ by prop $3.18, \mathrm{~F}_{\mathrm{A}}$ is not a fuzzy soft dense set in $(\mathrm{U}, \mathrm{E}, \psi)$. Hence $(\mathrm{U}, \mathrm{E}, \psi)$ is not a fuzzy soft hyper connected space .

\section{REFERENCE}

[1] K. K. Azad, On Fuzzy Semi-Continuity, Fuzzy Almost Continuity And Fuzzy Weakly Continuity, J. Math. Anal. Appl. 82(1981), 14-32.

[2] G. Balasubramanian, Fuzzy Disconnectedness and its Fuzzy Stronger Forms, Indian J. Pure Appl. Math., 24(1) (1993).

[3] C. L. Chang, Fuzzy Topological Spaces, J. Math. Anal. Appl. 24 (1968), 182-190.

[4] E. K. Van Douwen, Applications Of Maximal Topologies, Topo. And Appl., 51 (1993), 125-139.

[5] Maji, P.K., Roy, A.R., Biswas, R, 2001. Fuzzy Soft Sets. Journal Of Fuzzy Mathematics 9 (3), 589-602.

[6] Molodtsov, D, 1999. Soft Set Theory First Results. Comput. Math. Appl. 37, 19-31.

[7] On Fuzzy Soft Weak Baire Spaces E. Poongothai, s. Divyapriya .Advances in mathematics: scientific journal 9 (2020), no.8, 5945-5952 issn: 1857-8365 (printed); $1857-8438$ (electronic) https://doi.org/10.37418/amsj.9.8.62.

[8] On Fuzzy Soft Nowhere Dense Sets E Poongothai, S Divyapriya International journal of scientific research https://www.worldwidejournals.com/internationaljournal-of-scientific-research-(IJSR)/article/on-fuzzysoft-nowhere-dense-sets/MjUwMTQ=/. Volume-9 Issue-4 | April-2020 | PRINT ISSN No. 2277 - 8179 | DOI: 10.36106/ijsr.
[9] G.Thangaraj And S.Muruganantham On Fuzzy Perfectly Disconnected spaces International Journal Of Advances In Mathematics Volume 2017, Number 5, Pages 12-21, 2017.

[10] Roy, S., Samanta, T.K, 2013. An introduction to open and closed sets on fuzzy soft topological spaces. Ann. Fuzzy Math. Inform., 6 (2), 425-431.

[11] Tanay, B., Kandemir, M.B. 2011.Topological structure of fuzzy sets comput. Math.Appl., 61(10), 2952-2957.

[12] L. A. Zadeh, Fuzzy Sets, Inform. And Control,8(1965), 338-353. 